International Journal of Quantum Information

Vol. 11, No. 8 (2013) 1399001 (8 pages)

(c) World Scientific Publishing Company

DOI: $10.1142 /$ S0219749913990013

\title{
Author Index Volume 11 (2013)
}

Abdel-Aty, M., see Kadry, H.

$11(2013) 1350038$

Abdel-Khalek, S. and Almalki, M. S., Entanglement for Jaynes

Cummings Model in the Presence Multi-Photon Process Under

Decoherence Effect

$11(2013) 1350026$

Agarwal, S. and Hashemi Rafsanjani, S. M., Maximizing Genuine Multipartite Entanglement of $N$ Mixed Qubits

Ahl Laamara, R., see Daoud, M.

Albayrak, E., The Entangled Quantum Heat Engine in the Various Heisenberg Models for a Two-Qubit System

Almalki, M. S., see Abdel-Khalek, S.

Ashourisheikhi, S. and Sirsi, S., Local Unitary Equivalent Classes of Symmetric N-Qubit Mixed States

Attia, M., see Chatta, R.

Bahrampour, A. R., see Jahanshahi, S.

Banaszek, K., see Lasota, M.

Batle, J., Plastino, A., Plastino, A. R. and Casas, M., Discord-

Entanglement Interplay in the Thermodynamic Limit: The

$X Y$-Model

Ben-Av, R., see Exman, I.

Boettcher, S., see Lara, P. C. S.

Casas, M., see Batle, J.

Chatta, R., Gueddana, A. and Attia, M., Realizability Assessment of Probabilistic Quantum CNOT Gate Based on Experimental

Implementation

$11(2013) 1350032$

Cheng, W., Xu, F., Li, H. and Wang, G., Entanglement of Convex

Linear Combination and Construction of PPT Entangled States

Chen, A.-X., see Zhang, J.-S.

Chen, J.-L., see $\mathrm{Su}, \mathrm{H} .-\mathrm{Y}$.

Chen, S., see $\mathrm{Li}$, J.

Chen, X.-B., see Wei, Z.-H.

Chen, X.-B., Yang, S., Xu, G., Su, Y. and Yang, Y.-X., Cryptanalysis of the Quantum State Sharing Protocol Using Four Sets of W-Class States

Chen, X.-Y., see Jiang, L.-Z.

Cheong, L. Y., see Kadry, H.

Chiang, C.-F., Quantum Phase Estimation with an Arbitrary Number of Qubits

11 (2013) 1350043

$11(2013) 1350057$

11 (2013) 1350021

11 (2013) 1350026

11 (2013) 1350072

11 (2013) 1350032

11 (2013) 1350020

11 (2013) 1350034

11 (2013) 1350003

11 (2013) 1350028

11 (2013) 1350069

11 (2013) 1350003

11 (2013) 1350002

11 (2013) 1350058

11 (2013) 1350019

11 (2013) 1350066

11 (2013) 1350068

11 (2013) 1350010

11 (2013) 1350060

11 (2013) 1350038

11 (2013) 1350008 
Daoud, M., Ahl Laamara, R. and Essaber, R., Quantum Correlations Dynamics of Quasi-Bell Cat States

de Assis, F. M., see Guedes, E. B.

Debarba, T., see Marchiolli, M. A.

Demkowicz-Dobrzański, R., see Lasota, M.

Deng, D.-L., see Su, H.-Y.

Dong, H.-K., see Dong, L.

Dong, L., Wang, J.-X., Xiu, X.-M., Dong, H.-K., Li, D. and Gao, Y.-J., A Generation Scheme of the Distributed Four-Photon Cluster-Type Polarization-Entangled States Exploiting the

Integration of Entanglement Gates and the Controlled Phase Gate

Du, G., see Liu, B.

$\mathrm{Du}, \mathrm{R} .-\mathrm{G}$. , see Sun, Z.-W.

El Allati, A., Hassouni, Y. and Metwally, N., Quantum

Communication via Heisenberg XYZ System

Essaber, R., see Daoud, M.

Exman, I. and Ben-Av, R., Remote Scalable Entangling of Symmetric Dicke States

11 (2013) 1350057

11 (2013) 1350022

11 (2013) 1330001

$11(2013) 1350034$

$11(2013) 1350019$

11 (2013) 1350064

$11(2013) 1350064$

11 (2013) 1350018

11 (2013) 1350005

$11(2013) 1350035$

11 (2013) 1350057

$11(2013) 1350028$

Ezerman, M. F., Jitman, S., Kiah, H. M. and Ling, S., Pure Asymmetric Quantum MDS Codes from CSS Construction: A Complete Characterization

Fang, A., see Wang, Y.

Fang, J., Lu, Y., Huang, P., He, G. and Zeng, G., Discretely Modulated Continuous-Variable Quantum Key Distribution with a Nondeterministic Noiseless Amplifier

Fan, H., see Jia, L.-X.

Farooq, K., see Khan, M. A.

Farooq, K., see Zhang, X. Y.

Feng, Y., see Liu, Y.

Furman, G. B., Meerovich, V. M. and Sokolovsky, V. L., Zero Field Entanglement in Dipolar Coupling Spin System at Negative Temperature

Galetti, D., see Marchiolli, M. A.

Ganti, A. and Somma, R., On the Gap of Hamiltonians for the Adiabatic Simulation of Quantum Circuits

Gao, S., see Wang, Y.

Gao, Y.-J., see Dong, L.

Gawron, P., see Winiarczyk, R.

Gawron, P., Kurzyk, D. and Puchała, Z., A Model for Quantum Queue

Gou, L., see Liu, B.

Gueddana, A., see Chatta, R.

Guedes, E. B. and de Assis, F. M., On the Security of DecoherenceFree Subspaces and Subsystems for Classical Information Conveying Through Quantum Channels
$11(2013) 1350027$

11 (2013) 1350040

11 (2013) 1350037

11 (2013) 1350048

11 (2013) 1350017

11 (2013) 1350046

11 (2013) 1350006

11 (2013) 1350050

11 (2013) 1330001

11 (2013) 1350063

11 (2013) 1350040

11 (2013) 1350064

11 (2013) 1350007

11 (2013) 1350023

11 (2013) 1350018

11 (2013) 1350032

11 (2013) 1350022 
Guenda, K. and Gulliver, T. A., Symmetric and Asymmetric Quantum Codes

$11(2013) 1350047$

Gulliver, T. A., see Guenda, K.

Guo, Y., Sun, X. and Zhang, W., An Arbitrated Quantum Signature

Scheme Based on Hyperchaotic Quantum Cryptosystem

Hashemi Rafsanjani, S. M., see Agarwal, S.

Hassouni, Y., see El Allati, A.

He, G. P., Simple Quantum Protocols for the Millionaire Problem with a Semi-Honest Third Party

He, G., see Fang, J.

Hong, F.-Y., see Jiang, L.-Z.

Hou, S. C., see Khan, M. A.

Hou, X.-W., see Li, X.-J.

Huang, P., see Fang, J.

Huang, Z.-Y., see Zhang, J.-S.

Huo, M.-X., Li, Y., Song, Z. and Sun, C.-P., A Quantum Simulator for Probing Mott Lobes via the AC Josephson Effect

Hwang, M.-R., see Jung, E.

Hwang, T., see Yang, C.-W.

Jahanshahi, S., Bahrampour, A. R. and Zandi, M. H., Security Enhanced Direct Quantum Communication with Higher Bit-Rate

Ji, H.-H., see Li, X.-J.

Ji, Y.-Q., see Jin, Z.

Jia, L.-X., Li, B., Yue, R.-H. and Fan, H., Sudden Change of Quantum Discord Under Single Qubit Noise

Jiang, L.-Z., Chen, X.-Y., Ye, T.-Y., Hong, F.-Y. and Wu, L.-N., Entanglement Criterion for Coherent Subtraction and Coherent Addition of Bipartite Continuous Variable States

11 (2013) 1350047

$11(2013) 1350036$

11 (2013) 1350043

11 (2013) 1350035

11 (2013) 1350025

11 (2013) 1350037

11 (2013) 1350060

11 (2013) 1350017

11 (2013) 1350070

11 (2013) 1350037

11 (2013) 1350058

11 (2013) 1350049

11 (2013) 1350061

11 (2013) 1350052

11 (2013) 1350020

11 (2013) 1350070

11 (2013) 1350055

11 (2013) 1350048

11 (2013) 1350060

Jin, Z., Ji, Y.-Q., Su, S.-L., Si, B., Wang, H.-F., Zhang, S. and Zhu, A.-D., Nondestructive Entanglement Analysis and Generation with Atoms in Low-Q Cavities Assisted by Coherent Light

Jitman, S., see Ezerman, M. F.

Jung, E., Hwang, M.-R. and Park, D., Quantum Discord and Quantum Entanglement in the Presence of an Asymptotically Flat Static Black Hole

Jurkowski, J., Quantum Discord Derived from Tsallis Entropy

Kadry, H., Zakaria, N., Cheong, L. Y. and Abdel-Aty, M., Dynamical Properties of Multi-Photon Interaction Between a Cavity Field and a Single-Qubit

Khan, M. A., Hou, S. C., Farooq, K. and Yi, X. X., Single Photon Reflection and Transmission in Optomechanical System

$11(2013) 1350027$

11 (2013) 1350061

$11(2013) 1350013$

$11(2013) 1350038$

$11(2013) 1350017$

11 (2013) 1350027

11 (2013) 1350023

11 (2013) 1350016

Lai, X.-B., see Li, S.-S.

Lara, P. C. S., Portugal, R. and Boettcher, S., Quantum Walks on Sierpinski Gaskets

$11(2013) 1350069$ 
Lasota, M., Demkowicz-Dobrzański, R. and Banaszek, K., Quantum Key Distribution with Realistic Heralded Single-Photon Sources

Li, B., see Jia, L.-X.

Li, C.-K., Roberts, R. and Yin, X., Decomposition of Unitary Matrices and Quantum Gates

Li, D., see Dong, L.

Li, F., see Wang, Y.

$\mathrm{Li}, \mathrm{H}$. , see Cheng, W.

Li, J., Li, L., Chen, S. and Liu, X., A New Detection Strategy for Two-Step QSDC Protocol Based on the Five-Particle Cluster State

11 (2013) 1350034

11 (2013) 1350048

$11(2013) 1350015$

11 (2013) 1350064

11 (2013) 1350040

11 (2013) 1350002

$11(2013) 1350066$

11 (2013) 1350066

11 (2013) 1350040

11 (2013) 1350006

11 (2013) 1350016

11 (2013) 1350070

11 (2013) 1350049

11 (2013) 1350027

11 (2013) 1350018

11 (2013) 1350054

11 (2013) 1350011

11 (2013) 1350044

11 (2013) 1350066

11 (2013) 1350011

11 (2013) 1350006

11 (2013) 1350011

11 (2013) 1350005

11 (2013) 1350037

11 (2013) 1350042

11 (2013) 1350044

11 (2013) 1350053

11 (2013) 1350033

11 (2013) 1350042

11 (2013) 1350006

11 (2013) 1350009

11 (2013) 1330001 
McKague, M., On the Power Quantum Computation Over Real Hilbert Spaces

$11(2013) 1350001$

Meerovich, V. M., see Furman, G. B.

11 (2013) 1350050

Metwally, N., see El Allati, A.

Miszczak, J. A., see Winiarczyk, R.

Mo, Z.-W., see Peng, J.-Y.

Mo, Z.-W., see Peng, J.-Y.

Mosk, A. P., see Škorić, B.

11 (2013) 1350035

11 (2013) 1350007

11 (2013) 1350004

11 (2013) 1350044

11 (2013) 1350041

Murao, M., see Nakata, Y.

11 (2013) 1350062

Nakata, Y. and Murao, M., Diagonal-Unitary 2-Design and Their

Implementations by Quantum Circuits

Niu, X.-X., see Wei, Z.-H.

$11(2013) 1350062$

11 (2013) 1350068

11 (2013) 1350019

11 (2013) 1350012

11 (2013) 1350061

Park, D., see Jung, E.

Park, D., Entanglement Degradation in the Presence of $(4+n)$-Dimensional Schwarzschild Black Hole

$11(2013) 1350014$

Pavlyukh, Y. and Rau, A. R. P., 1-, 2-, and 6-Qubits, and the Ramanujan-Nagell Theorem

Pawela, Ł., see Winiarczyk, R.

Peng, J.-Y., Luo, M.-X., Mo, Z.-W. and Liu, H.-W., Flexible Deterministic Joint Remote State Preparation of Some States

Peng, J.-Y. and Mo, Z.-W., Hierarchical and Probabilistic Quantum State Sharing with a Nonmaximally Four-Qubit Cluster State

Petulante, N., see Liu, C.

Ping, Y., Zhang, C., Wang, M. and Wang, S., Output Entanglement in a Four-Level Correlated Emission Laser

Pinkse, P. W. H., see Škorić, B.

Pinto, E., Trindade, M. A. S. and Vianna, J. D. M., Quasitriangular Hopf Algebras, Braid Groups and Quantum Entanglement

Plastino, A. R., see Batle, J.

Plastino, A., see Batle, J.

Portugal, R., see Lara, P. C. S.

Prudêncio, T., Quantum State Transfer and Hadamard Gate for Coherent States

Puchała, Z., see Winiarczyk, R.

Puchała, Z., see Gawron, P.

Qin, S.-J., see Zuo, H.-J.

Rau, A. R. P., see Pavlyukh, Y.

Renes, J. M., The Physics of Quantum Information:

Complementarity, Uncertainty, and Entanglement

Roberts, R., see Li, C.-K.

$11(2013) 1350056$

11 (2013) 1350007

$11(2013) 1350044$

11 (2013) 1350004

11 (2013) 1350054

11 (2013) 1350071

11 (2013) 1350041

11 (2013) 1350065

$11(2013) 1350003$

11 (2013) 1350003

11 (2013) 1350069

11 (2013) 1350024

11 (2013) 1350007

11 (2013) 1350023

11 (2013) 1350030

11 (2013) 1350056

$11(2013) 1330002$

$11(2013) 1350015$

Sadowski, P., Generating Efficient Quantum Circuits for Preparing Maximally Multipartite Entangled States

11 (2013) 1350067 
Shchesnovich, V. S., Asymptotic Evaluation of Bosonic Probability Amplitudes in Linear Unitary Networks in the Case of Large Number of Bosons

$11(2013) 1350045$

Shojaei, S., Mahdian, M. and Yousefjani, R., Electric Field Effects on Quantum Correlations in Three Coupled Semiconductor Quantum Dots

Si, B., see Jin, Z.

Sirsi, S., see Ashourisheikhi, S.

Škorić, B., Mosk, A. P. and Pinkse, P. W. H., Security of QuantumReadout PUFs Against Quadrature-Based Challenge-Estimation Attacks

Sokolovsky, V. L., see Furman, G. B.

Somma, R., see Ganti, A.

Song, H., Pan, Y. and Xi, Z., Dynamical Control of Quantum Correlations in a Common Environment

Song, H.-S., see Wu, S.-X.

Song, T.-T., see Zuo, H.-J.

Song, Z., see Huo, M.-X.

Su, H.-Y., Chen, J.-L., Wu, C., Deng, D.-L. and Oh, C. H., Detecting Einstein-Podolsky-Rosen Steering for Continuous Variable Wavefunctions

$11(2013) 1350009$

11 (2013) 1350055

11 (2013) 1350072

$11(2013) 1350041$

11 (2013) 1350050

11 (2013) 1350063

$11(2013) 1350012$

11 (2013) 1350029

11 (2013) 1350030

11 (2013) 1350049

$\mathrm{Su}$, S.-L., see Jin, Z.

$\mathrm{Su}, \mathrm{Y}$., see Chen, X.-B.

Sun, C.-P., see Huo, M.-X.

Sun, C., see Liu, B.

Sun, X., see Guo, Y.

Sun, Z.-W., Du, R.-G. and Long, D.-Y., Quantum Key Distribution with Limited Classical Bob

Tang, P., see Ma, S.-Y.

Teng, J. H., Ma, J. and Wang, W., The Spectra and Optical Bistability of Cavity Field Coupled to a Mechanical Mirror

Trindade, M. A. S., see Pinto, E.

Vianna, J. D. M., see Pinto, E.

Wang, C., Xu, G. and Yang, Y.-X., Cryptanalysis and Improvements for the Quantum Private Comparison Protocol Using EPR Pairs

Wang, G., see Liu, B.

Wang, G., see Cheng, W.

Wang, H.-F., see Jin, Z.

Wang, J.-X., see Dong, L.

Wang, M., see Ping, Y.

Wang, S., see Ping, Y.

Wang, W., see Teng, J. H.

$11(2013) 1350019$

11 (2013) 1350055

11 (2013) 1350010

11 (2013) 1350049

11 (2013) 1350018

11 (2013) 1350036

11 (2013) 1350005

11 (2013) 1350042

11 (2013) 1350033

11 (2013) 1350065

11 (2013) 1350065

11 (2013) 1350039

11 (2013) 1350018

11 (2013) 1350002

11 (2013) 1350055

11 (2013) 1350064

11 (2013) 1350071

11 (2013) 1350071

11 (2013) 1350033

Wang, Y., Gao, S., Fang, A., Li, P. and Li, F., Realization of Fast Quantum Information Transfer and Entanglement with Superconducting Flux Qubits Coupled to a Resonator

$11(2013) 1350040$ 
Wei, Z.-H., Chen, X.-B., Niu, X.-X. and Yang, Y.-X., A Novel

Quantum Steganography Protocol Based on Probability

Measurements

$11(2013) 1350068$

Winiarczyk, R., Gawron, P., Miszczak, J. A., Pawela, Ł. and

Puchała, Z., Analysis of Patent Activity in the Field of Quantum

Information Processing

$\mathrm{Wu}, \mathrm{C}$. , see $\mathrm{Su}, \mathrm{H} .-\mathrm{Y}$.

$\mathrm{Wu}$, L.-N., see Jiang, L.-Z.

Wu, S.-X., Zhang, J., Yu, C.-S. and Song, H.-S., Quantum

Correlations in the Entanglement Distillation Protocols

$\mathrm{Xi}, \mathrm{Z}$., see Song, $\mathrm{H}$.

Xiu, X.-M., see Dong, L.

$\mathrm{Xu}, \mathrm{F}$., see Cheng, $\mathrm{W}$.

$\mathrm{Xu}, \mathrm{G}$. , see Chen, X.-B.

$\mathrm{Xu}, \mathrm{G}$. , see Wang, C.

Xue, K., see Liu, B.

Yang, C.-W. and Hwang, T., Efficient Key Construction on SemiQuantum Secret Sharing Protocols

Yang, S., see Chen, X.-B.

Yang, Y.-X., see Chen, X.-B.

Yang, Y.-X., see Wang, C.

Yang, Y.-X., see Wei, Z.-H.

Ye, L., see Zhu, M.-Z.

Ye, T.-Y., see Jiang, L.-Z.

Ye, T.-Y., Large Payload Bidirectional Quantum Secure Direct

Communication Without Information Leakage

Yi, X. X., see Khan, M. A.

Yi, X. X., see Zhang, X. Y.

Yin, X., see Liu, D.

Yin, X., see Li, C.-K.

Yousefjani, R., see Shojaei, S.

$\mathrm{Yu}$, C.-S., see Wu, S.-X.

Yue, R.-H., see Jia, L.-X.

Zakaria, N., see Kadry, H.

Zandi, M. H., see Jahanshahi, S.

Zeng, G., see Fang, J.

Zhang, C., see Ping, Y.

Zhang, J., see Wu, S.-X.

Zhang, J.-S., Huang, Z.-Y. and Chen, A.-X., Bipartite and

Multipartite Correlations of Coupled Qubits in a Non-Markovian

Environment: Hierarchy Equation Method

$11(2013) 1350058$

Zhang, S., see Jin, Z.

Zhang, W., see Guo, Y.

11 (2013) 1350055

11 (2013) 1350036

Zhang, X. Y., Farooq, K. and Yi, X. X., Instantaneous Dark State and Trajectory Tracking

$11(2013) 1350046$ 
Zhang, Z., see Liu, D.

$11(2013) 1350011$

Zhu, A.-D., see Jin, Z.

11 (2013) 1350055

Zhu, M.-Z. and Ye, L., Implementation of Swap Gate and Fredkin Gate Using Linear Optical Elements

$11(2013) 1350031$

Zuo, H.-J., Qin, S.-J. and Song, T.-T., Discussion on Quantum Proxy Group Signature Scheme with $\chi$-Type Entangled State

11 (2013) 1350030 